Meta

Journal des traducteurs

Translators' Journal

\title{
Aphasie et traduction
}

\section{Michel Paradis}

Volume 29, numéro 1, mars 1984

Cerveau, langage et traduction

URI : https://id.erudit.org/iderudit/003781ar

DOI : https://doi.org/10.7202/003781ar

Aller au sommaire du numéro

Éditeur(s)

Les Presses de l'Université de Montréal

ISSN

0026-0452 (imprimé)

1492-1421 (numérique)

Découvrir la revue

Citer cet article

Paradis, M. (1984). Aphasie et traduction. Meta, 29(1), 57-67.

https://doi.org/10.7202/003781ar

Ce document est protégé par la loi sur le droit d'auteur. L'utilisation des services d'Érudit (y compris la reproduction) est assujettie à sa politique d'utilisation que vous pouvez consulter en ligne.

https://apropos.erudit.org/fr/usagers/politique-dutilisation/
Cet article est diffusé et préservé par Érudit.

Érudit est un consortium interuniversitaire sans but lucratif composé de l’Université de Montréal, l'Université Laval et l'Université du Québec à Montréal. Il a pour mission la promotion et la valorisation de la recherche. https://www.erudit.org/fr/ 


\title{
APHASIE ET TRADUCTION
}

\author{
Michel PARADIS
}

Un très petit nombre d'observations relatives à la traduction chez des aphasiques ${ }^{1}$ bilingues ont été rapportées au cours des 150 dernières années. La raison de cette lacune découle vraisemblablement du fait que, d'une part, il n'existait aucun examen standard pour évaluer la nature et l'étendue des troubles du langage chez les aphasiques bilingues et que, d'autre part, les auteurs qui vérifiaient la connaissance résiduelle de chaque langue n'examinaient pas systématiquement les capacités de traduction chez leurs malades.

Charcot (1887) signale un patient qui, alors qu'il parlait cinq langues couramment avant son aphasie, avait perdu la capacité de s'exprimer en français (apparemment sa langue maternelle) autrement qu'en traduisant au préalable de l'allemand ou de l'espagnol. De même, Kauders (1929) rapporte le cas d'un patient qui, lorsqu'il éprouvait de la difficulté à évoquer les mots en allemand, sa langue maternelle, les trouvait par l'intermédiaire de leur équivalent dans la langue étrangère. À plusieurs reprises, une patiente de Denès (1914) traduisit spontanément en français les mots et les phrases qu'on lui faisait lire, vraisemblablement dans le but d'indiquer qu'elle en comprenait le sens. Parfois elle répondait en français à des questions posées en italien mais n'était pas capable de traduire ses réponses en italien. Veyrac (1931) mentionne une patiente écholalique qui à deux reprises traduisit, automatiquement et sans donner aucun signe de compréhension, deux phrases de l'anglais, sa langue maternelle peu usitée depuis des années, au français, sa langue devenue la plus familière. Une patiente de Goldstein (1948) passait (de manière d'ailleurs assez inappropriée) d'une langue à l'autre mais était incapable de traduire sur demande, alors qu'elle en était fort capable avant son aphasie. Jakobson (1964) rapporte qu'à la suite d'un accident d'automobile il avait été aphasique pendant deux heures environ au cours desquelles il ne pouvait s'empêcher de traduire, automatiquement et sans aucune nécessité, chacune de ses phrases en quatre ou cinq langues. Enfin l'Hermitte, Hécaen, Dubois, Culioli et Tabouret-Keller (1966) semblent avoir vérifé les capacités de traduction de leur 8 patients mais ne fournissent des exemples dans les deux sens que d'un seul patient, et une phrase chez un autre patient. Ils concluent néanmoins que d'une manière générale la traduction volontaire amplifie les difficultés spécifiques au type d'aphasie du patient.

Devant ces maigres données, pour la plupart anecdotiques, il s'avéra nécessaire d'élaborer, au sein d'un examen de l'aphasie chez les bilingues, un test de traduction. C'est ainsi que, en collaboration avec l'unité de recherche en neuropsychologie et neurolinguistique (U-111) de l'Institut national de la santé et de la recherche scientifique à $\mathrm{Pa}$ ris, alors sous la direction du professeur Henry Hécaen, l'équipe de recherche sur l'aphasie chez les bilingues du département de linguistique de l'université McGill à Montréal a mis au point un instrument de mesure des capacités de traduction chez les aphasiques. Il s'insère dans un examen qui comprend trois parties : (a) une partie com-

1. Un aphasique est un patient qui manifeste des troubles du langage consécutifs à une atteinte cérébrale. La nature de ces troubles varie généralement en fonction du site de la lésion. 
mune à toutes les langues, comprenant l'anamnèse, l'histoire détaillée du bilinguisme du patient et un examen neuropsychologique destiné à mettre en évidence les déficits associés (orientation dans le temps et dans l'espace, mémoire à court terme, mémoire des faits récents et anciens, praxies ${ }^{2}$, gnosies ${ }^{3}$ ); (b) un examen particulier à chacune des langues (langage spontané, discrimination auditive verbale, désignation d'objets, compréhension, répétition, dénomination, récitation de séries automatisées (jours de la semaine, compter jusqu'à 23) génération de phrases, test de fluence verbale, transformations grammaticales, exercices morphologiques et sémantiques, description d'une bande dessinée non humoristique, lecture, écriture, calcul mental) ; (c) un examen particulier à une paire de langues donnée (traduction, jugements d'acceptabilité de phrases incorporant des structures syntaxiques de l'autre langue).

Les versions de l'examen en différentes langues ne sont pas de simples traductions les unes des autres, mais des transpositions de manière à ce que chaque sous-test soit d'une complexité linguistique équivalente à celle de sa contrepartie dans toutes les langues. Dans certains cas, cette similitude sera entre la longueur des mots et leur fréquence d'utilisation (tel que dans l'épreuve de répétition de mots); les groupes consonantiques courants dans une langue sont remplacés par des groupes consonantiques différents, mais également courants dans l'autre, et les logatomes ${ }^{4}$ sont construits selon les règles phonologiques de chaque langue ; dans d'autres cas, la similarité sera entre la longueur de la phrase et son type structural (comme dans les épreuves de répétition de phrase), la longueur de la phrase et sa complexité linguistique (comme dans les épreuves de compréhension syntaxique ; une phrase contenant un verbe passif bien moins complexe dans une langue donnée qu'en français ou en anglais, avec la simple addition d'un préfixe par exemple, une structure différente de complexité comparable dans cette langue sera alors utilisée, par exemple une construction clivée) ; ou encore le contenu informatif et la structure de la phrase (comme dans la lecture d'un passage dont on doit par la suite fournir le contenu). Dans ce dernier cas, le rappel de l'information peut être mesuré par le nombre d'items retrouvés (le même nombre d'items de la même sorte : par exemple " un homme est allé à la chasse avec son fils " devient "une femme est allée à la pêche avec sa fille" dans une autre langue). Même structure de phrase, même nombre d'items à rappeler, bien que les items eux-mêmes (homme, aller à la chasse, fils/femme, aller à la pêche, fille) soient différents, de manière à éviter la facilitation du rappel de l'information contenue dans l'épreuve de l'autre langue subie la veille.

Un domaine où la différence est la plus évidente est dans le test de discrimination auditive verbale. Dans ce test, une série de quatre mots ne différant que par leur consonne initiale ou groupe consonantique initial, [et chaque fois qu'il est possible par un seul trait phonétique, par exemple $\mathrm{t} / \mathrm{d}, \mathrm{s} / \mathrm{z}, \mathrm{3} / \mathrm{s}$ (voisement) ou $\mathrm{d} / \mathrm{g}, \mathrm{t} / \mathrm{k}$ (point d'articulation)], sont représentés par une image. Un des mots est lu à haute voix et le patient doit montrer l'image qui, parmi les quatre, correspond au mot. Dans certaines langues, de telles paires minimales sont rendues impossibles par la structure phonologique de la

2. Coordination volontaire des mouvements vers le but proposé. Si le patient est incapable de faire le geste de montrer du doigt, il ne sera pas possible, par exemple, de vérifier sa compréhension en lui demandant de montrer du doigt l'image qui correspond à l'illustration de la phrase prononcée par l'examinateur. Son échec pourrait être dû́ tout aussi bien à son apraxie qu'à son manque de compréhension.

3. Faculté de reconnaître, par l'un des sens, la forme d'un objet, de se le représenter et d'en saisir la signification. Si le patient est incapable de reconnaître le sens d'une image, il ne sera pas possible, par exemple, de vérifier sa compréhension en lui demandant de montrer du doigt l'image qui correspond à une phrase prononcée par l'examinateur. Son échec pourrait être dû tout aussi bien à son agnosie visuelle qu'à son manque de compréhension.

4. Pseudo-mot construit selon les règles phonologiques de la langue testée, mais sans signification conventionnelle (exemples français : chafon, sivolure, lube). 
langue, qui n'admet pas par exemple de consonnes initiales voisées. Ceci élimine la possibilité de paires minimales basées sur le voisement/dévoisement de la consonne initiale. Par conséquent, dans de tels cas, l'opposition devra porter sur la consonne médiane ou finale de certains mots. Une contrainte supplémentaire est que le mot doit être facilement représentable par une image (ce qui élimine la plupart des mots abstraits) puisque le patient indique sa compréhension en montrant l'image correcte parmi trois distracteurs phonologiquement semblables (par ex. : main, nain, pain, bain).

Parfois les éléments d'une épreuve ont dû être adaptés de manière à tenir compte de certaines contraintes spécifiques à une langue donnée. Dans le test de Pierre Marie ${ }^{5}$, par exemple, les trois papiers ont été remplacés par trois clefs de tailles croissantes dans la version arabe, à cause des structures linguistiques impliquées dans la formulation de la consigne. Également dans la version arabe, la négation morphologique, trop régulière et donc inintéressante, a été remplacée par des alternations masculin/féminin impliquant tantôt une métathèse, tantôt un infixe, tantôt un suffixe. Et ainsi de suite pour les autres langues.

Les phrases du test de traduction ont été choisies de manière à maximiser le contraste syntaxique entre les deux langues. Par exemple, de l'anglais au français on trouve des phrases telles que she was given a watch for her birthday - on lui a donné une montre pour son anniversaire (au Québec : sa fête), où une phrase à la voix passive devient active avec un sujet indéfini ; her brother swam across the river - son frère a traversé la rivière à la nage, où une préposition devient le verbe et le verbe un complément de manière, et l'adjectif possessif s'accorde avec le possédé et non le possesseur ; my neighbour has been working in Paris for 2 years - mon voisin travaille à Paris depuis 2 ans, où le temps du verbe change, de même que la préposition; et ainsi de suite.

Grâce à un tel outil d'évaluation des capacités linguistiques du sujet bilingue, des comportements fort intéressants et pour le moins paradoxaux ont été mis en évidence. Paradis, Goldblum et Abidi (1982) décrivent en effet deux cas d'aphasiques bilingues qui, au cours d'une restitution antagoniste alternative de leurs deux langues étaient incapables de traduire de la langue qu'ils comprenaient fort bien à celle qu'ils comprenaient et parlaient sans difficulté ce jour-là alors qu'ils étaient capables de traduire correctement et sans hésitation vers la langue qui leur était momentanément inaccessible pour l'expression spontanée ou toute autre tâche linguistique.

Le premier cas est celui d'une infirmière née au Maroc de parents français, ayant appris l'arabe littéraire à l'école française et l'arabe dialectal par la suite, de manière intensive, à l'âge de 21 ans. Depuis l'âge de 24 ans, et jusqu'à son accident survenu à l'âge de 48 ans, elle parlait arabe avec ses malades et les aides infirmières, et français avec les médecins et ses proches. Un soir, à la sortie de son travail, elle fut renversée de son vélomoteur par une automobile, se heurta le crâne contre la chaussée et perdit connaissance pendant un quart d'heure. À son réveil à l'hôpital elle était totalement aphasique. Ce n'est que 4 jours plus tard qu'elle fut capable de prononcer quelques mots en arabe seulement, à la grande surprise de ses amies avec qui elle ne parlait que français. Lorsqu'on lui demanda pourquoi elle parlait arabe, elle répondit qu'elle ne pouvait pas faire autrement. Cette perte sélective du français dura quatre jours.

5. L'épreuve des " trois papiers" de Pierre Marie consiste en une consigne à trois étapes, énoncée en une seule fois. On remet au patient 3 papiers de tailles différentes et on lui demande : "Donnez-moi le petit, mettez le moyen sur vos genoux, jetez le grand ". Les résultats obtenus au test de mémoire à court terme et à l'examen des praxies permettent de juger dans quelle mesure un comportement déviant par rapport à la consigne peut être attribué à un trouble de la compréhension. 
Transférée à Paris 11 jours après l'accident, la patiente était lucide et s'exprimait en français. Le test administré en français 4 jours après son arrivée révéla une légère aphasie amnésique ${ }^{6}$. La patiente s'exprimait normalement, bien que son discours fût entrecoupé de pauses et d'hésitations provoquées par un manque de mot. Le lendemain, par contre, son français se révéla excessivement pauvre. Elle était incapable de décrire une série d'images. Son discours spontané était extrêmement réduit par manque de vocabulaire alors que son arabe était très bon. Le lendemain c'était le contraire : son arabe était très pauvre tandis que son français était redevenu tout à fait normal. Le jour suivant, elle parlait arabe de nouveau couramment. Elle quitta alors l'hôpital.

Ce qui nous paraît plus particulièrement intéressant, c'est que les jours où la patiente parlait arabe sans difficulté mais était incapable de parler français, elle était néanmoins capable de traduire de l'arabe au français, langue dans laquelle elle ne pouvait trouver ses mots pour dénommer les objets usuels qu'on lui présentait ou pour s'exprimer spontanément, alors qu'elle était tout à fait incapable de traduire du français, qu'elle n'avait jamais cessé de comprendre parfaitement, vers l'arabe qu'elle parlait tout à fait couramment ce jour-là. Le lendemain, sa traduction du français vers l'arabe était parfaite, alors qu'elle ne pouvait s'exprimer spontanément en arabe, et sa traduction de l'arabe au français était très mauvaise, alors qu'elle s'exprimait couramment en français ce jour-là. Huit jours plus tard, alors que son français était normal mais qu'elle ne trouvait pas ses mots en arabe, sa traduction de l'arabe au français était très bonne, tandis que sa traduction du français à l'arabe était très mauvaise. Deux semaines plus tard, alors que son arabe était redevenu presque aussi bon que son français, la traduction fut très mauvaise dans les deux sens.

Peu de temps après, à Montréal, un patient de 23 ans qui parlait couramment le français, sa langue maternelle, et l'anglais, sa langue de travail qu'il avait acquise avant même d'aller à l'école, manifesta un type semblable de récupération de ses deux langues à la suite d'une intervention chirurgicale. Pendant la première semaine qui suivit l'opération il ne pouvait parler que l'anglais, au point que son père devait servir d'interprète quand il s'adressait à sa femme. La deuxième semaine, il avait récupéré l'usage de son français mais ne pouvait plus s'exprimer en anglais. Au cours de la troisième semaine, lorsque le test de traduction lui fut administré, son anglais était en voie d'amélioration mais son français était néanmoins supérieur. Il traduisit correctement et sans hésitation les 6 phrases du français (sa langue la plus forte) à l'anglais (sa langue la plus faible) mais fut incapable de traduire les phrases de l'anglais (qu'il comprenait parfaitement) au français (qu'il parlait très couramment). Pour de plus amples détails le lecteur voudra bien consulter Paradis et coll. (1982).

Nous nous trouvons donc en présence de deux patients qui, à plusieurs reprises, ont été capables de traduire de la langue qui leur était la plus accessible vers la langue dans laquelle ils ne pouvaient s'exprimer spontanément par manque de capacité à évoquer les mots, ce qui semble d'autant plus paradoxal qu'ils étaient alors incapables de faire l'inverse, c'est-à-dire de traduire vers la langue dans laquelle ils n'avaient pas de difficulté à évoquer les mots pour s'exprimer spontanément. Pourtant, on suppose généralement que la version est plus facile que le thème. Ellis et Hennelly (1980) ont montré que la traduction vers la langue la mieux maîtrisée est plus rapide, et donc plus facile, que la traduction vers la langue la plus faible. Selon Goldstein (1948:142), on peut s'attendre à ce qu'un patient puisse traduire de la langue la plus atteinte vers la langue la

6. Trouble du langage généralement consécutif à une lésion cérébrale de la partie postérieure du lobe temporal gauche, caractérisé par une difficulté à évoquer les mots. Le manque du mot est en général le plus sévère lorsqu'on demande au sujet de dénommer des objets ou des images d'objets. Pour plus de détails, le lecteur voudra bien consulter Lecours, Lhermite et collaborateurs (1979), pp. 141-142. 
mieux préservée, mais pas le contraire. Or c'est précisément cette tendance contraire qu'ont manifestée nos deux patients.

On pourrait en conclure que dans le contexte d'une aphasie amnésique le patient parvient à évoquer le mot qui lui manque par le biais de la traduction et que le mot dans la langue inaccessible est recouvré grâce à l'évocation du mot équivalent dans la langue accessible. Cependant ce phénomène ne s'est pas produit de manière systématique. En effet, la traduction a été une fois possible dans la direction opposée, c'est-à-dire de la langue inaccessible vers la langue accessible, et une autre fois la traduction était impossible dans les deux sens alors que les deux langues étaient disponibles. Il semble donc raisonnable de conclure que la capacité de traduire est neuropsychologiquement indépendante de la capacité de parler deux langues.

On dit d'une personne qu'elle est bilingue lorsqu'elle est capable d'exprimer à volonté un message soit dans une langue, soit dans l'autre, en fonction des circonstances, c'est-à-dire de la langue de l'interlocuteur ou, dans le cas d'un interlocuteur également bilingue (ou de verbalisation à soi-même), du sujet, de la situation, de ce qui a immédiatement précédé ou de ce qui va suivre, ou de la présence de tierces personnes. Cependant, une fois qu'une telle personne bilingue a décodé un message dans une langue, il lui est souvent difficile d'encoder le même message immédiatement après dans l'autre langue. Vildomec $(1963: 54,214,238)$ fait remarquer que la maîtrise d'une deuxième langue n'implique pas nécessairement la capacité de traduire de l'une à l'autre ; que cette capacité doit être établie séparément, et qu'elle dépend entre autres de la formation et de l'occupation de la personne concernée. Pick $(1913$ : 132) soulignait déjà les difficultés considérables que même les bilingues parfaitement compétents dans les deux langues pouvaient éprouver à la traduction orale directe. C'est en effet très souvent le cas, sauf lorsque la personne a été amenée à énoncer souvent un même message successivement dans les deux langues. Il s'agit alors d'une capacité acquise implicitement ou apprise consciemment, celle de traducteur (ou plutôt d'interprète). En plus de son habileté à comprendre et à parler deux langues, un tel sujet aura acquis ou appris séparément et indépendamment, à donner des équivalents de l'une à l'autre ; séparément, c'est-à-dire en dehors de l'acquisition de l'une ou de l'autre langue, à des moments et par des moyens différents ; indépendamment, c'est-à-dire sans rapport de cause à effet avec cette acquisition dans un sens ou dans l'autre (acquisition - traduction ou traduction - acquisition). Cet apprentissage de la traduction est d'un ordre différent. Sachant déjà manier séparément chaque langue, le sujet apprend à produire sur demande des équivalents (de L1 à L2 et/ou de L2 à L1). Cette compétence en traduction rapide sera limitée aux expressions effectivement souvent répétées.

La difficulté qu'éprouve le bilingue (qui par ailleurs manipule parfaitement les deux langues) à produire sur demande une expression équivalent d'une langue à l'autre peut provenir de trois sources non mutuellement exclusives, la première d'ordre linguistique, les deux autres d'ordre neurophysiologique. Une expression dans une langue peut ne pas avoir d'équivalent rapproché dans l'autre et il est alors tout simplement difficile de rendre dans une langue ce qui a été énoncé dans l'autre sans circonlocutions compliquées. Mais ce n'est pas toujours le cas. Souvent il existe une expression plus ou moins équivalente et par ailleurs fort courante, et cependant l'individu bilingue n'arrive pas à la fournir immédiatement. Il semble même parfois que plus il la cherche, plus il s'acharne, plus l'expression équivalente devient inaccessible, pour faire surface spontanément quelques instants ou quelques heures plus tard. Ce phénomène peut s'expliquer neurophysiologiquement au moins de deux manières.

On peut concevoir que, lorsque le locuteur utilise une de ses deux langues, l'autre est automatiquement inhibée de manière à éviter les interférences. C'est un phénomène 
fort courant en neurophysiologie : toute activité antagoniste est inhibée concurremment à l'activation d'une fonction. L'évocation d'un mot chez l'unilingue peut s'expliquer non seulement par l'activation du substrat cérébral qui lui correspond mais concurremment par l'inhibition des systèmes sous-jacents de tous les autres champs sémantiques d'abord, des autres mots à l'intérieur d'un même champ ensuite, jusqu'à l'inhibition des circuits qui sous-tendent tous les mots à l'exclusion de celui qui est activé. Il est raisonnable de penser que le même principe s'applique à l'utilisation de deux langues : L2 est inhibée lors de l'utilisation de L1 et particulièrement l'expression en L1 équivalente de celle utilisée en $\mathbf{L} 2$. Ce principe peut également servir à expliquer les récupérations sélectives, successives et antagonistes (q.v. dans Paradis et Lecours 1979) : la langue inaccessible de manière permanente ou temporaire n'est pas détruite mais inhibée, une solution déjà proposée par Pitres (1895).

La traduction orale immédiate (interprétation) implique la violation du principe de séparation des deux langues par l'inhibition de la langue ou des langues autre(s) que celle qui est momentanément utilisée. Il semble difficile de chercher une phrase équivalente dans une langue pendant que l'on garde en mémoire à court terme, c'est-à-dire de manière consciente, la phrase à traduire (c'est-à-dire la forme aussi bien que le sens). Une stratégie efficace pour surmonter cet inconvénient serait de débarrasser la mémoire à court terme de la phrase de la langue source de manière à ne retenir que son message (c'est-à-dire le sens dépouillé de sa forme) afin de pouvoir encoder ce message dans la langue cible sans être gêné par l'inhibition que cause l'activation de la phrase source, ni par l'interférence qui consiste à chercher des équivalents mot à mot ou syntagme par syntagme au lieu d'encoder la force illocutoire du message dans une forme appropriée à la langue cible.

Une deuxième manière d'expliquer la difficulté qu'éprouve un bilingue à traduire d'une langue à l'autre, surtout dans les cas pathologiques, alors qu'il était fort capable et même avait l'habitude de traduire avant son aphasie, serait par l'inhibition des circuits qui sous-tendent spécifiquement les connexions établies par la pratique d'une expression à son équivalent dans l'autre langue.

En effet, au moins deux stratégies sont accessibles à l'interprète.

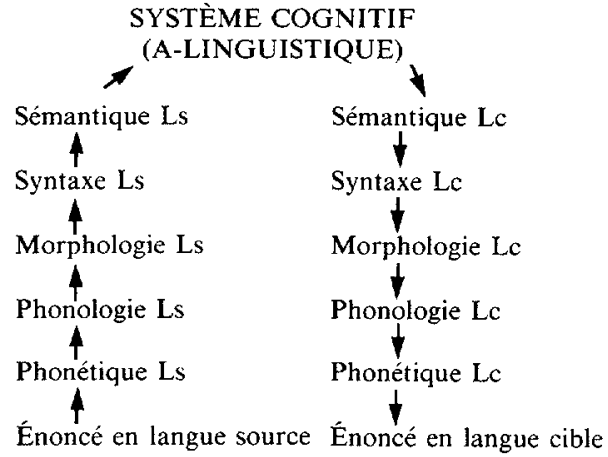

Stratégie I : Décodage linguistique dans la langue source jusqu'à la compréhension du message (conscience du sens), suivi de

\section{SYSTÈME COGNITIF}

(A-LINGUISTIQUE)

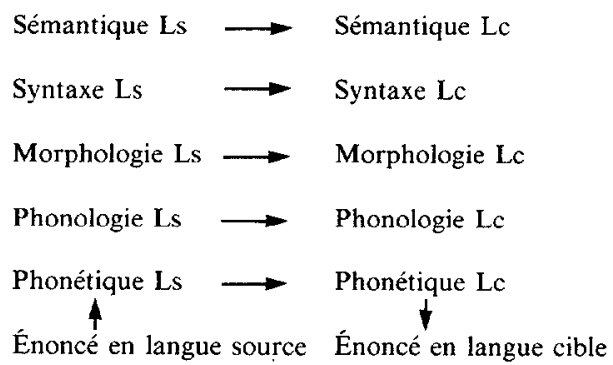

Stratégie II : Transcodage direct par application machinale de règles, d'un élément de la langue source à un élément équivalent 
l'encodage linguistique du message dans la langue cible.

\begin{abstract}
de même niveau dans la langue cible. Ce type de transcodage peut se faire à chaque niveau : aux niveaux phonétique et phonologique pour la prononciation des noms propres, par exemple; au niveau morphologique (par ex. : anglais -ical $\rightarrow$ français -ique); syntaxique (par ex. : anglais I have been V-ing for... $\rightarrow$ français $J$ e $V$ (présent) depuis); lexical (par ex. : décevoir $\rightarrow$ disappoint); sémantique (par ex. : parents $\rightarrow$ (famille) relatives (père et mère) parents).
\end{abstract}

Il peut soit décoder l'énoncé de la langue source jusqu'à l'obtention du sens du message pour l'encoder ensuite dans la langue cible de la manière la plus appropriée, soit passer directement de l'énoncé en langue source à son équivalent (appris et répété) en langue cible. Dans le premier cas, comme nous l'avons vu, alors que la phrase (en tant que phrase, c'est-à-dire forme + sens) est maintenue en mémoire à court terme, et donc que les traces neurales sous-jacentes aux expressions de la langue source (y compris leur forme phonologique) sont activées, on peut supposer que les expressions équivalentes dans la langue cible sont par la même occasion inhibées. En outre, chaque fois que la force illocutoire d'un énoncé de la langue source doit être réalisée par un énoncé de type différent dans la langue cible, ou simplement par une phrase de structure syntaxique différente comme c'est le cas non seulement des proverbes et des expressions idiomatiques et stéréotypées, mais encore d'expressions non idiomatiques qui se traduisent néanmoins par des phrases de structure tout à fait différente), la phrase retenue en mémoire à court terme (entre autres sous sa forme phonologique) incite à chercher des mots équivalents, des expressions équivalentes, voire des phrases équivalentes. Une manière d'éliminer l'inhibition serait donc de se débrarrasser en mémoire à court terme de la forme acoustique de l'énoncé en langue source. D'une part, dans la mesure où la structure linguistique de la langue source serait écartée, la structure équivalente de la langue cible ne serait plus inhibée et deviendrait donc plus facilement accessible, et d'autre part la tendance à traduire au niveau de la structure de surface plutôt que de rendre le même message selon les conventions propres à la langue cible serait surmontée. Dans le deuxıème cas, il s'établirait un lien entre une expression dans une langue et son équivalent dans l'autre. Ce lien serait une sorte de court-circuit qui éviterait le décodage complet de l'énoncé en langue source de même que les décisions à l'encodage du message dans la langue cible. Ainsi de nouvelles connexions s'établiraient entre le substrat neural d'une expression dans une langue et celui de son équivalent dans l'autre. Ces connexions seraient indépendantes de celles qui sous-tendent les systèmes linguistiques proprement dits et pourraient donc être indépendamment activées de même qu'indépendamment inhibées par la condition pathologique. En effet, le patient montréalais mentionné dans Paradis et coll. (1982), quand on lui demandait de désigner le plafond, la fenêtre, la porte, ou le lit (sur lequel il était assis), fournissait spontanément le mot équivalent dans l'autre langue, mais était incapable de montrer la porte, le plafond ou le lit. Il déclarait avoir l'assurance que ces objets existaient dans la pièce, mais était incapable de les identifier, ce qui ne l'empêchait pas d'en donner la traduction. Il pouvait donc produire la traduction de certains mots sans être capable de se rappeler à quoi ils se réfé- 
raient. Ce comportement montre que la capacité de traduire peut exister en dehors de la compréhension du sens référentiel des mots traduits. La malade écholalique de Veyrac (1931) avait déjà fourni un exemple de ce comportement en traduisant des phrases en l'absence d'accès à l'une ou l'autre langue, ni pour la compréhension, ni pour l'expression spontanée. D'autre part, Paradis et coll. (1982) rapportent le cas d'incapacité de traduction alors que les deux langues étaient accessibles aussi bien pour la compréhension que pour l'expression.

L'apprentissage des raccourcis d'une structure de phrase ou d'une expression d'une langue vers sa traduction dans l'autre permet de passer directement de la phrase ou du syntagme à la phrase ou au syntagme équivalent. Dans cette optique, plus les équivalents seront souvent rapprochés, plus les traces neurofonctionnelles sous-jacentes seront activées, plus la traduction sera facile. Chaque occurrence de traduction aurait pour effet de réduire l'impact de l'inhibition en établissant d'abord, et en renforçant ensuite, une nouvelle connexion entre équivalents d'une langue à l'autre. En d'autres termes, chaque traduction d'une expression à une autre aurait pour effet de baisser le seuil d'excitation de la trace qui lie les deux expressions et par conséquent de rendre la prochaine traduction plus facile. Les expressions souvent traduites seraient donc plus facilement accessibles que les expressions moins souvent traduites.

Ces connexions entre les éléments équivalents des deux langues pourraient expliquer pourquoi les patients de Paradis et coll. (1982) étaient capables d'accéder à la langue cible par le biais de la traduction alors que cette langue était inaccessible directement : La condition pathologique ayant inhibé cette langue, c'est-à-dire en ayant haussé le seuil d'excitation au-delà de la limite permettant l'auto-activation des traces, tout en permettant leur activation à partir de stimuli extérieurs (compréhension), permettrait également leur activation par l'intermédiaire des connexions entre l'expression de la langue source (la langue forte) et celle de la langue cible (la langue faible). Cela n'explique cependant pas pourquoi les mêmes patients étaient incapables de traduire de la langue faible (qu'ils comprenaient parfaitement) à la langue forte qu'ils pouvaient parler spontanément. Il faudrait alors supposer que, lorsqu'une langue n'est pas accessible pour l'expression spontanée (évocation ou auto- activation), c'est-à-dire lorsque le seuil d'excitation des traces est trop élevé pour pouvoir évoquer les éléments linguistiques en l'absence de stimuli extérieurs, les connexions qui vont d'une expression de cette langue faible à son équivalent dans la langue forte (qui est accessible) ne sont pas non plus fonctionnelles, bien que le stimulus (la phrase source) soit donné (et compris). Toutefois, l'accès à la langue source ne saurait être une condition suffisante puisque certains patients ayant accès à leurs deux langues se sont montrés incapables de traduire (Goldstein 1948 ; Paradis et coll. 1982, cas 1). D'autre part, à une occasion, la patiente de Paradis et coll. (1982) a été capable de traduire de la langue faible vers la langue forte et l'inverse. La capacité de traduire d'une langue à l'autre semble donc tout à fait indépendante de l'accessibilité de l'une ou l'autre langue pour fins d'expression spontanée.

Il semble donc que 3 systèmes neurofonctionnels soient impliqués dans la traduction simultanée (interprétation) d'une phrase : le système sous-tendant la langue source, celui sous-tendant la langue cible, et celui sous-tendant les connexions de la langue-cible à la langue-source. Un quatrième système est impliqué lorsque langue-cible et languesource sont interverties. Nous avons vu en effet que la traduction de L1 à L2 était possible alors que celle de $\mathbf{L} 2$ à $\mathbf{L} 1$ ne l'était pas, chez un même patient. La traduction repose donc sur deux systèmes de connexions, un pour chaque direction, puisqu'ils sont susceptibles de dysfonctionnement sélectif. Ces quatre systèmes semblent neurofonctionnellement autonomes bien que reliés entre eux et intégrés dans leur fonctionnement chez le sujet bilingue normal. Toutefois chacun de ces quatre systèmes semble capable 
d'inhibition sélective de même que de fonctionnement isolé, en l'absence du fonctionnement des autres systèmes.

L'inhibition de la langue autre que celle que l'on est en train d'utiliser ne bloque cependant pas sa compréhension. En fait il est même impossible au bilingue de choisir de ne pas comprendre les énoncés qu'il perçoit quelles que soient les circonstances. Cette constatation a amené certains auteurs à se demander s'il n'y avait pas deux systèmes sous-jacents : l'un sous-tendant la compréhension, l'autre sous-tendant l'expression (voir Albert et Obler 1978 : 220). Plusieurs arguments peuvent être avancés en faveur d'une telle supposition : La compréhension précède l'expression au cours des premières étapes du développement du langage chez l'enfant; la compréhension d'une langue étrangère dépasse la capacité de s'exprimer dans cette langue ; lorsqu'une langue n'est plus utilisée, l'expression se détériore plus vite que la compréhension; en particulier chez les aphasiques amnésiques, la compréhension est récupérée avant l'expression ; certains aphasiques bilingues conservent la compréhension d'une langue qui leur est inaccessible pour l'expression.

Cependant, ces phénomènes peuvent s'expliquer plus économiquement si on suppose un seul système de traces sous-jacentes avec un seuil d'activation différent pour la compréhension et l'expression. Goldstein (1948), souscrivant à l'opinion de Bastian (1898), note que, suite aux atteintes les plus sévères, le cerveau ne réagit à aucun stimulus ; que dans les cas un peu moins sévères il ne réagit qu'aux stimulations sensorielles directes; que enfin il réagit à toute stimulation sauf volontaire (c'est-à-dire autostimulation), et que, dans ce dernier cas, le patient est capable de comprendre et de répéter ce qu'il est incapable de produire spontanément (c'est-à-dire volontairement). De même, selon un principe bien établi en psychologie, la reconnaissance est plus facile que l'évocation (Piaget $1970: 245$ ). Sur le plan linguistique, la reconnaissance correspond à la compréhension de l'énoncé, l'évocation (ou rappel) correspond à l'auto-activation volontaire des structures linguistiques nécessaires à l'expression, en l'absence de stimuli extérieurs. L'inhibition peut avoir pour effet de hausser le seuil d'activation d'une trace, ce qui permettrait de comprendre malgré tout la langue inhibée tout en étant incapable de l'évoquer volontairement. Ce principe peut expliquer également le fait qu'une langue qui n'a pas été utilisée depuis des années soit comprise encore longtemps après qu'il soit devenu impossible de s'exprimer dans cette langue, que la compréhension soit récupérée avant l'expression dans les aphasies amnésiques, et que les aphasiques bilingues puissent comprendre une de leurs langues sans y avoir accès pour s'exprimer. L'expression volontaire qui implique l'auto-activation des traces requiert une trace plus robuste (c'està-dire une trace activée plus souvent, ou plus récemment, ou établie avec une forte participation émotive, dont le seuil d'activation est par conséquent plus bas) que celle nécessaire à la compréhension, et il suffit de postuler un seul système de traces soustendant à la fois les aspects réceptifs et expressifs de la langue.

Il n'en va pas de même en ce qui concerne les traces sous-jacentes aux traductions d'une langue à l'autre et vice versa puisque chaque direction peut être inhibée isolément et que tantôt la traduction est possible seulement dans un sens, tantôt seulement dans l'autre chez un même patient à l'intérieur d'un espace de temps assez limité (de 24 heures à une semaine). Il semble donc que les traces dans chaque direction soient neurofonctionnellement séparées, établies séparément, et susceptibles d'inhibition sélective. De plus, leur activation ne semble pas déterminée par la disponibilité de la langue source ou de la langue cible.

En résumé, un très petit nombre de cas de traduction chez les aphasiques a été rapporté jusqu'ici. Afin de pallier ce manque de données, un test de traduction a été incorporé à l'examen mis au point pour détecter les différences de performance possibles 
dans chacune des langues de l'aphasique bilingue. L'utilisation expérimentale de ce test a révélé des comportements de traduction paradoxaux. À différents moments, une patiente a démontré qu'il était possible de comprendre et de parler deux langues sans pouvoir traduire de l'une à l'autre quelle que soit la direction, qu'il était possible de traduire de $\mathbf{L} 1$ à $\mathbf{L} 2$ mais non de $\mathbf{L} 2$ à $\mathbf{L} 1$, même lorsque $\mathbf{L} 1$ était accessible pour l'expression volontaire et $\mathbf{L} 2$ ne l'était pas. Deux patients ont montré qu'il était possible de traduire sans comprendre. Nous en avons déduit que la traduction pouvait, dans certains cas, être neurofonctionnellement indépendante de la capacité de comprendre et de parler deux langues.

Nous avons considéré deux stratégies de traduction : (1) le décodage du message de la langue source suivi de l'encodage du message dans la langue cible en passant par un stade non linguistique de représentation cognitive du message, et (2) l'établissement de connexions directes entre éléments de $L 1$ et leurs équivalents en $\mathrm{L} 2$ et vice versa. Pour être efficace, il semble que la première stratégie doive s'accompagner de la capacité d'oblitérer en la mémoire à court terme la forme acoustique de l'énoncé de manière à éviter l'inhibition de l'expression en langue cible (tout en évitant la tendance à traduire mot à mot ou expression par expression). La seconde stratégie a pour effet de contourner l'inhibition de la langue cible en utilisant une voie autre que celle de l'évocation volontaire habituelle, fournissant une source de stimulation différente. Cette route indirecte est proposée comme tentative d'explication des comportements de traduction paradoxaux observés chez des aphasiques bilingues au cours d'une récupération antagoniste alternative de leurs deux langues. Contrairement à ce que nous avons vu à propos des différences de performance entre la compréhension et l'expression (lesquelles peuvent s'expliquer par une différence de seuil d'activation), il ne suffit pas de postuler une seule et même trace de la L1 à la L2 et de la L2 à la L1 puisque chacune est susceptible d'inhibition sélective aussi bien que de fonctionnement isolé.

Nous sommes donc en mesure de conclure que les connexions établies entre expressions équivalentes peuvent être indépendantes de celles qui sous-tendent les langues elles-mêmes. Il semble y avoir 4 systèmes neurofonctionnels indépendants : un système sous-tendant $\mathrm{L} 1$, un deuxième système sous-tendant $\mathrm{L} 2$, un troisième système soustendant les connexions entre équivalents de la $\mathrm{L} 1$ à la $\mathrm{L} 2$, et un quatrième système soustendant les connexions entre équivalents de la L2 à la L1. Toutefois, dans la mesure où le bilingue est capable de décoder de la langue source et de réencoder dans la langue cible sans passer par le raccourci qui lie les équivalents les uns aux autres, il est capable de traduire sans faire appel à un système sous-jacent spécifique à la traduction. C'est probablement la stratégie du traducteur occasionnel, non habitué, qui a coutume de parler chaque langue avec un groupe de locuteurs différent. Par contre, l'interprète professionnel a probablement établi des connexions entre équivalents qui lui permettent de contourner l'inhibition de la langue cible. De toute façon, l'autonomie neurofonctionnelle de la traduction semble établie : la compréhension de deux langues peut exister sans capacité de traduire et la capacité de traduire peut exister sans compréhension des 2 langues. Il est à espérer qu'à l'avenir l'utilisation systématique de l'instrument de mesure que nous avons mis au point apportera des éléments permettant une analyse plus approfondie du phénomène neuropsychologique complexe que représente la traduction simultanée.

\section{RÉFÉRENCES}

ALBERT, M.L. et L.K. OBLER (1978) : The Bilingual Brain, New York, Academic Press. BASTIAN, H.C. (1898) : Treatise on Aphasia and other Speech Defects, London, Lewis.

CHARCOT, J.M. (1887) : Oeuvres completes de J.M. Charcot, t. III, A. Delahaye \& E. Lacrossier. 
DENÈS, P. (1914) : Contribution à l'étude de quelques phénomènes aphasiques, Paris, Hospice général de Nantes.

ELLIS, N.C. et R.A. HENNELLY (1980) : "A Bilingual Word-length Effect : Implications for Intelligence Testing and the Relative Ease of Mental Calculation in Welsh and English", British Journal of Psychology, 71, pp. 43-51.

FLORĖS, C. (1970) : "Mémoire à court terme et mémoire à long terme", dans B. BOVET et coll., la Mémoire, Paris, PUF.

GOLDSTEIN, K. (1948) : Language and Language Disturbances, New York, Grune \& Stratton.

JACOBSON, R. (1964) : "General Discussion", dans A.V.S. DE REUCK et Maeve O'CONNOR (eds.), Disorders of Language, Boston, Little, Brown, pp. 116-121 et pp. 248-250.

KAUDERS, O. (1929) : "Über polyglotte Reaktionen bei einer sensorischen Aphasie", Zeitschrift für die gesamte Neurologie und Psychiatrie, 122: 651-666.

L'HERMITTE, R., H. HÉCAEN, J. DUBOIS, A. CULIOLI et A. TABOURET-KELLER (1966) : "Le problème de l'aphasie des polyglottes : remarques sur quelques observations ", Neuropsychologia, 4, pp. 315-329.

PARADIS, M., M.-C. GOLDBLUM et R. ABIDI (1982) : "Alternate Antagonism with Paradoxical Translation Behavior in two Bilingual Aphasic Patients", Brain and Language, 15, pp. 55-69.

PARADIS, M. et A.R. LECOURS (1979) : "L'aphasie chez les bilingues et les polyglottes ", dans A.R. LECOURS, F. LHERMITTE et coll., l'Aphasie, Paris, Flammarion, pp. 605-616.

PIAGET, J. (1970) : "Mémoire et intelligence", dans B. BOVET et coll., la Mémoire, Paris, PUF.

PICK, A. (1913) : Die agrammatischen Sprachstörungen. Studien zur psychologischen Grundlegung der Aphasielehre, Berlin, Springer.

PITRES, A. (1895) : "Étude sur l'aphasie chez les polyglottes ", Revue de médecine, 15, pp. 873-899.

VEYRAC, G.-J. (1931) : Étude de l'aphasie chez les sujets polyglottes, thèse pour le doctorat en médecine, Université de Paris.

VILDOMEC, V. (1963) : Multilinguism, Leyden, Sythoff. 